\title{
Palmprint Recognition Based on Regional Rank Correlation of Directional Features
}

\author{
Yufei Han, Zhenan Sun, Tieniu Tan, and Ying Hao \\ Center for Biometrics and Security Research, National Laboratory of Pattern Recognition \\ Institute of Automation, Chinese Academy of Sciences \\ \{yfhan, znsun, tnt, yhao\}@nlpr.ia.ac.cn
}

\begin{abstract}
Automatic personal identification based on palmprints has been considered as a promising technology in biometrics family during recent years. In pursuit of accurate palmprint recognition approaches, it is a key issue to design proper image representation to describe skin textures in palm regions. According to previous achievements, directional texture measurement provides a powerful tool for depicting palmprint appearances. Most of successful approaches can be ranged into this framework. Following this idea, we propose a novel palmprint representation in this paper, which describes palmprint images by constructing rank correlation statistics of appearance patterns within local image areas. Promising experimental results on two large scale palmprint databases demonstrate that the proposed method achieves even better performances than the state-of-the-art approaches.
\end{abstract}

\section{Introduction}

Palmprint recognition technology distinguishes one individual from others based on differences of skin appearances in central palm regions of hands [1]. According to previous work, most of discriminating line-like image patterns in palmprints can be captured using only a low-resolution imaging device, like web cameras or low-cost CCD cameras. Due to its applicability, palmprint recognition has attracted increasing attention during recent years, especially in civil use, such as airport and custom.

In this paper, we focus on palmprint analysis based on low-resolution ( $<=100 \mathrm{dpi})$ images. In low resolution palmprint images, there are amount of line segments and irregularly distributed patches on skin surfaces, which are formed due to movement of fingers, structures of tissue and muscles after birth. They are discriminative patterns for personal identification task [1]. Early research in palmprint recognition borrows idea from fingerprint recognition [2][3]. There are many algorithms proposed to makes use of geometry information of principal lines, datum points, delta point features or even minutiae. However, they all require relatively high computation cost and are sensitive to appearance variations. In most state-of-the-art approaches [4-10], palmprints are treated as directional image textures spread in images, which are well characterized by a variety of local texture descriptors, such as wavelet coefficients [5], Gabor feature [1][7][8] and so on. Local texture analysis provides comprehensive and accurate descriptions for both spatial information and texture properties of palmprints. Thus it achieves the most efficient palmprint recognition by far. 
Motivated by the framework of local texture analysis, we propose to utilize correlation statistics of local distribution of texture features to represent palmprints. In our approach, directional DoG (Difference of Gaussian) filters are performed on palmprint images straightly to extract basic structures of palmprint appearances. Inside each local region, characteristics of skin textures are further encoded by spatial enhanced correlation statistics of distribution of the low-level texture descriptors, named regional appearance correlation (RAC) in this paper. This statistical representation describes distribution properties of skin textures accurately. Since the proposed correlation features does not lie on Euclidean space. Similarity between the proposed correlation based features is evaluated based on correlation matrix distance which is previously used in [14].

The remainder of this paper is organized as follows. Section 2 introduces the correlation based local texture descriptor. Section 3 describes details of the proposed RAC scheme. In Section 4, we demonstrate performances of the proposed approaches on two large-scale palmprint databases. Section 5 concludes the whole paper.

\section{Regional Appearance Correlation Based Palmprint Descriptor}

\subsection{Rank Correlation Statistics as Local Texture Descriptor}

In the proposed palmprint representation, we utilize the first and second order DoG filters (Differential of Gaussian) to extract information about structures of skin textures in palmprint images. In Eq.1 and Eq. 2, we illustrate the general expressions of the first and second order DoG filters $f^{\theta}$ and $g^{\theta}$ respectively, where $x_{1}=x \cos \theta+y \sin \theta, y_{1}=-x \sin \theta+y \cos \theta$

$$
\begin{aligned}
& f^{\theta}=-\frac{2 x_{1}}{\delta_{x}^{2}} \exp \left(-\left(\frac{x_{1}}{\delta_{x}}\right)^{2}-\left(\frac{y_{1}}{\delta_{y}}\right)^{2}\right) \\
& g^{\theta}=\left\{4 \frac{x_{1}^{2}}{\delta_{x}^{4}}-\frac{2}{\delta_{x}^{2}}\right\} \exp \left(-\left(\frac{x_{1}}{\delta_{x}}\right)^{2}-\left(\frac{y_{1}}{\delta_{y}}\right)^{2}\right)
\end{aligned}
$$

In Eq. 1 and Eq.2, $\theta$ is the rotation angle of the filters in 2D Cartesian coordinates. In scale space theory [11][12], DoG filters have been used as efficient indicators of differential image structures. The first order Gaussian derivate, also known as canny operator, is widely used for estimating image gradients and describing edges or contrast variations. The directional second order Gaussian derivate is close to optimal for detecting orientation of line-like patterns, like Gabor filters [13]. Benefited from Gaussian kernel, both of them can provide noise suppression in differentiating operation. In the proposed method, we perform convolution with DoG filters on palmprint images pixel by pixel. The size of the proposed filters is fixed to $35^{*} 35$. We set $\delta_{y} / \delta_{\mathrm{x}}=$ 3 for the scale to achieve good orientation selection. Empirical results testify that this setting is suitable for describing palmprints patterns. Furthermore, based on $\mathrm{X}-\mathrm{Y}$ separable property of Gaussian derivates [12], the 2D convolution procedure is computationally efficient. At each pixel, we can obtain two 6D filtering response vectors 
$v_{f}$ and $v_{g}$ containing filter results on six orientations $\{\theta=i \pi / 6(i=0,1 \ldots 5)\}$. The $i$ th components of $v_{f}$ and $v_{g}$ expose and enhance basic first and second order differential structures of palmprint appearances along the orientation $i \pi / 6$ respectively, which corresponds to directional edges and line-like segments in palm regions. Thus, the filtering response vectors $\left\{v_{f}\right\}$ and $\left\{v_{g}\right\}$ inside a local region form a comprehensive description of image structures along multiple orientations. In this paper, the proposed palmprint representation is designed based on distribution properties of the vectors over local regions, which can capture intrinsic texture characteristics of palmprint images in a statistical way.

In each local region, we firstly calculate rank correlation statistics of the $6 \mathrm{D}$ vectors $\left\{v_{f}\right\}$ and $\left\{v_{g}\right\}$ aggregated inside the region respectively to construct a local texture descriptor. This idea originates from use of covariance as a successful region descriptor in object recognition and detection [14]. From the aspect of image description, covariance statistics evaluate correlation between multiple image features, which achieves information fusion of different image descriptors. In our work, we firstly calculate covariance matrix $C O V$ of $k$ DD response vectors $\left\{v^{i}(i=1,2 \ldots \mathrm{k})\right\}(v=v f$ or $\left.v_{g}\right)$ inside a local block following Eq.3, where $\bar{v}$ is the average of : $\left\{v^{i}(i=1,2 \ldots \mathrm{k})\right\}$ :

$$
\operatorname{COV}_{i, j}=\frac{1}{k-1} \sum_{n=1}^{k}\left(v_{i}^{n}-\overline{v_{i}}\right)\left(v_{j}^{n}-\overline{v_{j}}\right)
$$

Nevertheless, as we can see in Eq.3, elements of covariance have close relation with the scalar value of the filtering responses that can be easily affected by appearance variations due to local contrast variations and deformation of skin surface in palm regions. As a result, it reduces robustness of covariance based feature against the appearance changes between intra-class samples and limits improvement of accuracy. To attack this problem, we propose to use spearman correlation (rank correlation) instead. Firstly, for each filtering vector $v_{f}$ or $v_{g}$ in a local region, we sort components of the vector in a descending order. Rank indexes of the six components form a rank statistics vector $S_{f}$ or $S_{g}$, corresponding to $v_{f}$ or $v_{g}$ respectively. This procedure is illustrated in Fig.1. After that, we compute a covariance matrix D on all rank statistics vectors $\left\{S_{f}\right\}$ or $\left\{S_{g}\right\}$ inside the region following Eq.3. Finally, a $6 * 6$ rank correlation matrix $R$ is then derived based on the covariance matrix $\mathrm{D}$, which is used as a local texture descriptor in the proposed method. Eq.4 illustrates the procedure:

$$
R=\left(\begin{array}{llll}
1 & r_{12} & \cdots & r_{16} \\
r_{21} & 1 & \cdots & r_{26} \\
\vdots & \vdots & & \vdots \\
r_{61} & r_{62} & \cdots & 1
\end{array}\right) \quad r_{i j}=\frac{d_{i j}}{\sqrt{d_{i i}} \sqrt{d_{j j}}}
$$

In Eq.4, dij is the corresponding elements of the covariance matrix D. In our scheme, we replace scalar filtering responses with their rank statistics. This method has been also known as ordinal encoding procedure [9]. For image representation, qualitative ordinal relation or rank statistics between image features presents robustness to local 
monotonous appearance variations or outlier observations. The ordinal feature reflects intrinsic structures of image patterns or descriptions. Notably, the rank statistics are widely used as discriminative indicators of directional skin textures in palm regions [7][8][9]. In the rank correlation matrix, non-diagonal elements are normalized correlation coefficients. Compared with covariance matrix, the normalized correlation coefficients remove affection of variation of original filtering results in a further step. They evaluate correlation measure between multiple components in the rank statistics vector, which is the intrinsic source of descriptive power in correlation based features. Furthermore, rank correlation coefficient is a non-parametric robust statistic. It doesn't require any prior assumption about function forms of multivariate distributions. As a matter of fact, in palmprint recognition, we seldom know about the exact form of distribution of local filtering responses. Thus, rank correlation provides more flexible tool to describe palmprint appearances than parametric statistics, such as covariance measure in Eq.3, which is not distribution-free.

$$
\begin{gathered}
\text { Original } \\
\text { filtering result }
\end{gathered}
$$

Fig. 1. Construction of rank statistics vector

\section{Construction of Regional Appearance Correlation}

Before further processing, we regulate geometrical deformation in palmprint images and crop square regions of interests (ROI) for feature extraction. To construct descriptions of local texture characteristics, we divide the whole ROI image into nonoverlapped local blocks with a specific spatial resolution, as illustrated in Fig.3. The texture description of the whole palmprint images is formed by rearranging the proposed statistical palmprint descriptors of each block, named Regional Appearance Correlation (RAC), according to spatial arrangement of the blocks.

Construction of the proposed RAC descriptor is shown in Fig.2. Each local block is further divided into four sub-regions. In the $i$ th sub-region, as described in Section.2, after convolution with the first and second order DoG filters at six orientations, we obtain $6 \mathrm{D}$ filtering response vectors $v_{f}$ and $v_{g}$ at each pixel. Then two $6 * 6$ rank correlation matrices $\mathbf{M} i$ and $\mathbf{M}_{i+5}$ are calculated based on all filtering response vectors $\left\{v_{f}\right\}$ and $\left\{v_{g}\right\}$ in this sub-block respectively. This procedure is shown in Fig.2. They indicate characteristics of distribution of local image structures in the $i$ th sub-region. Furthermore, we obtain correlation matrix M5 and M10, which are calculated based on all vectors $\left\{v_{f}\right\}$ and $\left\{v_{g}\right\}$ inside the whole local block respectively. Both of them evaluate global statistical texture properties in the block. In our method, they are used as complementation to correlation features that focus on finer texture structures within subregions. Thus, for each block in the image, we derive totally ten correlation matrices, which form a $6 * 6 * 10$ tri-tensor $\{\mathrm{M} i\}(i=1,2 \ldots, 9,10)$, named as Regional Appearance Correlation (RAC) in this paper. The first five matrices in this tri-tensor are derived based on all $\left\{v_{f}\right\}$ in this block, while left five are calculated from all $\left\{v_{g}\right\}$ in the block, 
as we can see in Fig.3. Based on the proposed scheme, we can find that the overall palmprint representation of the whole image involves spatial information of palmprint textures on two successive levels. For two RAC descriptor A and B derived from two separate local blocks, their orders of arrangement in the overall image representation denote geometric relation between two blocks, which represents topological structures of palmprint textures at block-level. Similarly, within each block, the precedence relation between different components $\mathrm{M} i$ in a RAC descriptor indicates spatial layout of palmprint image structures at finer sub-region level. Through this way, we can combine statistical texture descriptions on both coarse and fine spatial scales to achieve a comprehensive description of palmprint appearances within the local block. In our method, because the proposed correlation matrix doesn't lie on Euclidean space, we utilize a distance metric for symmetric correlation matrix as in Eq.5, which is proposed in [14]. Similarity between two RAC A and B is evaluated by calculating the mean of distance measures between corresponding $\mathrm{A} i$ and $\mathrm{B} i$ in the RAC descriptors.

$$
\operatorname{Dis}\left(A_{i}, B_{i}\right)=\sqrt{\sum_{k=1}^{n} \ln ^{2} \lambda_{k}\left(A_{i}, B_{i}\right)} \quad(i=1,2 \ldots 10)
$$

$\left\{\lambda_{k}\right\}$ are the generalized eigen values of $\mathrm{A} i$ and $\mathrm{B} i$. To testify efficiency of correlation based feature, we also construct RAC descriptor based on covariance matrices of original filtering results in our work, named as "covariance based RAC". Comparison of performances between two types of RAC descriptors is involved in the following section.

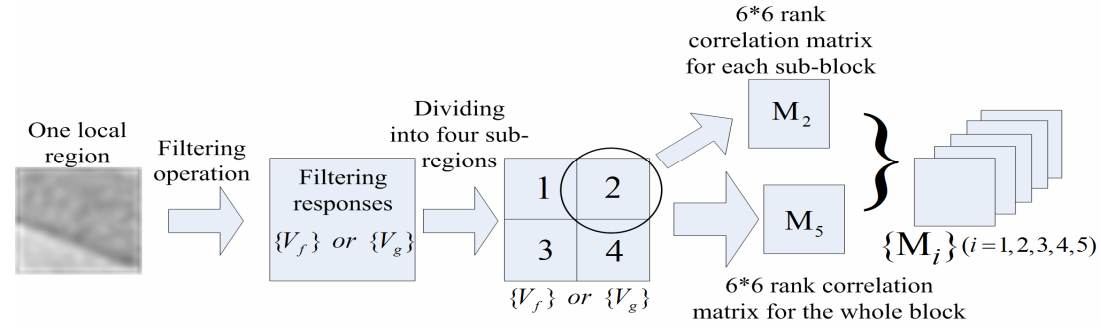

Fig. 2. Generation of rank correlation matrices inside a local image block

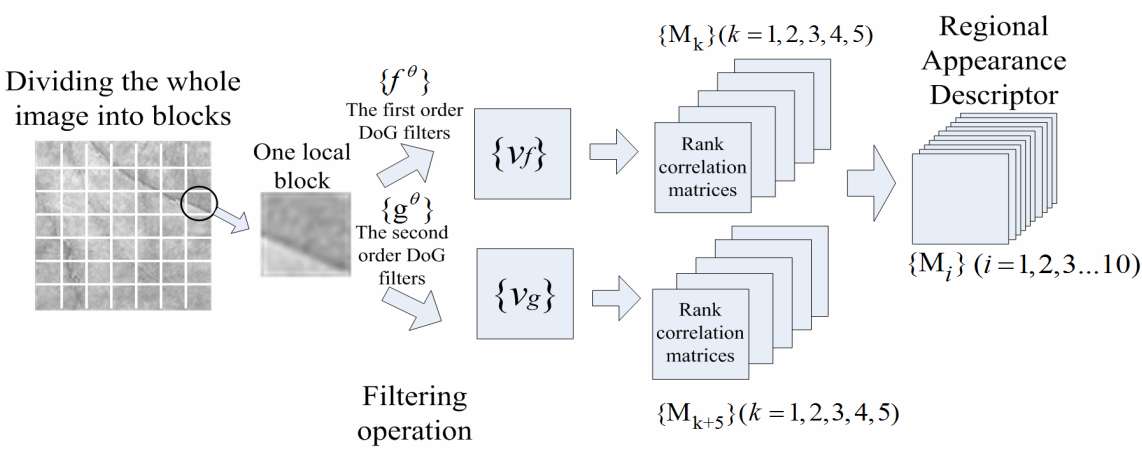

Fig. 3. Construction of Regional Appearance Descriptor 


\section{Experimental Results}

We test the correlation based representation on two large-scale palmprint databases, CASIA Palmprint Database [16] and PolyU Palmprint Database Version 2.0 [15]. Both of them contain deformation of skin textures due to variations of hand postures during image capturing, which is shown in Fig.4. In PolyU Palmprint Database Version 2.0 [15], each palm contain two different sessions. The average time interval between them is about two months. Light conditions and focus of the imaging device are changed between two occasions of image capture [1], which is challengeable to recognition algorithms. Palmprint images in CASIA Palmprint Database [16] are captured without pegs or plates to fix users' hands during image capturing [16]. Orientations and postures of hands are varied in the captured images, as we can see in Fig.7. It leads to contraction and stretching of skin surface in palm regions. In our experiments, we implement the other three state-of-the-art component based recognition algorithms [7][8][9] and evaluate performances on the two databases. The proposed correlation based features is compared with them to testify validity of the proposed method.

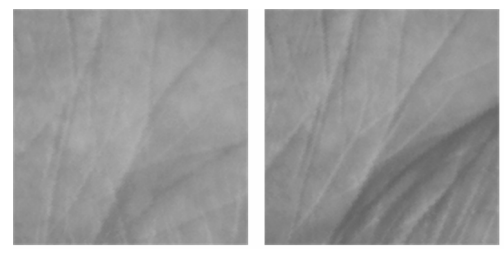

(a)
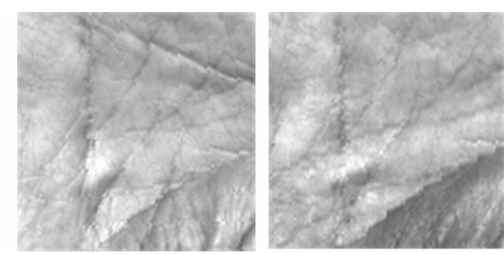

(b)

Fig. 4. Examples of deformation of skin textures in CASIA Palmprint Database [16] (a) and PolyU Palmprint Database [15] (b)

\subsection{Experimental Results on PolyU Palmprint Database Version 2.0}

There are totally 7,752 palmprint images from 385 palms in the PolyU database [15]. Six examples of captured images in this database are shown in Fig.5. After preprocessing, regions of interests, with the size of $128 \times 128$ are obtained from captured image. To construct the proposed RAC description, we divide the whole ROI into $16 * 16$ local blocks firstly. For each local region, we further construct the proposed RAC descriptor. Therefore, for each ROI image, we can obtain totally $8 * 8$ RAC descriptors which are arranged according to their spatial layouts to form appearance representation of the whole ROI image. Fig.6 and Tab.1 illustrate comparison of performances between our proposed algorithm and the other three state-of-the-art component based approaches [7][8][9]. As we can see in experimental results, we can find that our method achieves much lower EER than the state-of-the-art component based methods [7][8][9]. Furthermore, comparison between roc curves of RAC scheme and "RAC based on covariance" testifies that the local appearance variations result in deterioration of performances of covariance based feature, as we described in Section.2. The proposed rank correlation based texture description improves robustness to appearance change to a great extent. 
Table 1. Performances comparisons on PolyU Palmprint Database Version 2.0

\begin{tabular}{|c|c|l|}
\hline Algortihms & $d^{\prime}[17]$ & EER[17] \\
\hline RAC & 4.10 & $0.01 \%$ \\
\hline RAC based on covariance & 3.81 & $1.01 \%$ \\
\hline Ordinal Code [9] & 6.19 & $0.05 \%$ \\
\hline Competitive Code [8] & 5.84 & $0.04 \%$ \\
\hline Fusion code [7] & 5.40 & $0.21 \%$ \\
\hline
\end{tabular}
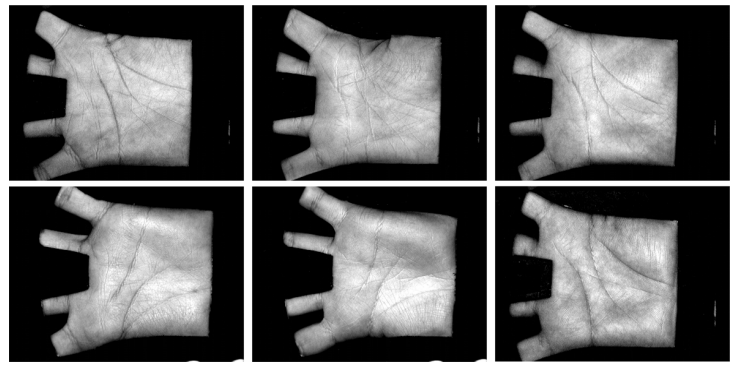

Fig. 5. Six examples of captured images of PolyU Palmprint Database Version 2.0

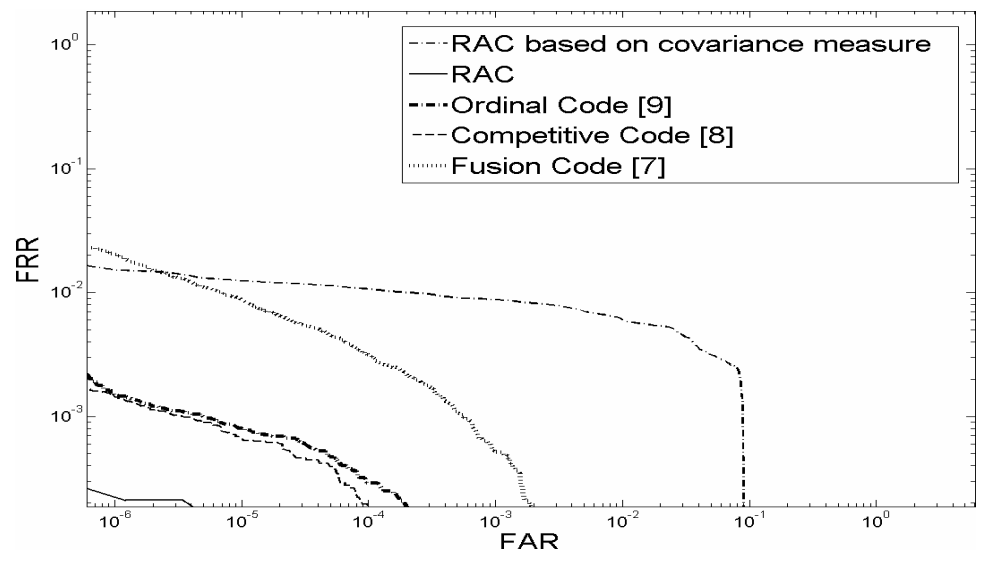

Fig. 6. ROC curves of all algorithms on PolyU Palmprint Database Version 2.0

\subsection{Experimental Results on CASIA Palmprint Database}

CASIA Palmprint Database contains totally 4,512 palmprint images from 282 palms which are captured with common cameras and peg-free palmprint imaging devices. The derived images are all 640*480 RGB color images [16], as shown in Fig.7. After transforming them into gray images and performing normalization procedure on them, $176 * 176$ ROI are obtained. For each ROI, we divide it into $22 * 22$ blocks. For each local block, we further sub-divide it into $11 * 11$ sub-regions and construct local RAC palmprint descriptors based on local blocks. As we can see in Fig.8 and Tab.2, we can draw similar conclusion that the rank correlation really extract stable and discriminative 
directional characteristics of palm textures, while covariance based scheme suffers from local appearance variations. It is worth to note that intra-class samples in this dataset are almost captured in a same time session. Thus, the CASIA database contains less appearance variations, such as changes of contrast and deformation of skin textures than the PolyU database, which are the main reasons for deterioration of performances of covariance based RAC scheme. Therefore, as we can see in the experimental results, the covariance based feature performs relatively better than the state-of-the-art approaches in the CASIA database.

Table 2. Performances comparisons on CASIA Palmprint Database

\begin{tabular}{|c|c|l|}
\hline Algortihms & $d^{\prime}[17]$ & EER[17] \\
\hline RAC & 4.77 & $0.03 \%$ \\
\hline RAC based on covariance & 5.69 & $0.05 \%$ \\
\hline Ordinal Code [9] & 5.65 & $0.08 \%$ \\
\hline Competitive Code [8] & 3.82 & $0.19 \%$ \\
\hline Fusion code [7] & 3.73 & $0.57 \%$ \\
\hline
\end{tabular}
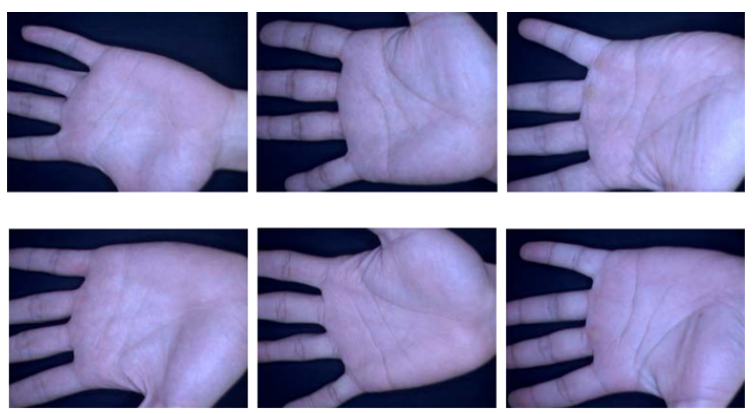

Fig. 7. Six examples of captured images of CASIA Palmprint Database

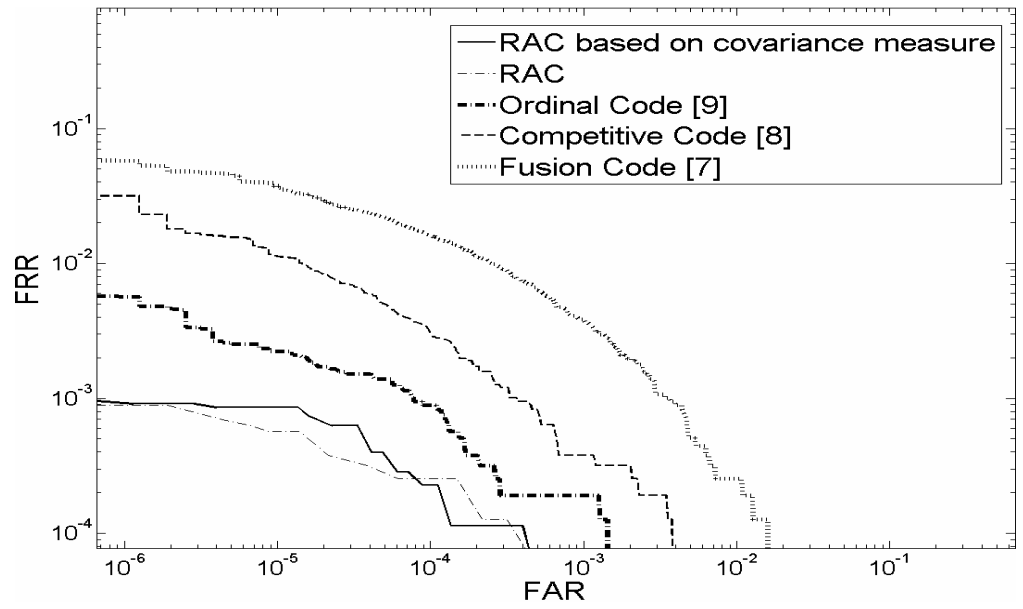

Fig. 8. ROC curves of all algorithms on CASIA Palmprint Database 


\section{Conclusion}

In this paper, we propose a rank correlation based palmprint image representation. It is composed by two main elements. We firstly divide the whole image to local blocks and enhance image structures within each block along multiple orientations using the first and second order directional DoG filter banks over the whole palmprint image. Characteristics of palmprints inside each local region are then evaluated by rank correlation statistics of filtering responses aggregated in the region, which actually form a description of non-isotropic image textures. By utilizing the rank correlation statistics, we obtain robustness to local appearance deformation due to qualitative coding of filtering responses. More importantly, the rank statistics extracts intrinsic characteristics of directional skin textures within local regions. In a further step, correlation measure between qualitative descriptions along different orientations provides accurate descriptions of local image structures. Experimental results on CASIA Palmprint Database [16] and PolyU Palmprint Database Version 2.0 [15] illustrate effectiveness of our method. Compared with the state-of-the-art methods [7][8][9], the proposed method achieves even higher accuracy. The proposed correlation measure based image descriptor can be applied not only in palmprint recognition, but also in face and iris image analysis in biometrics.

\section{Acknowledgement}

This work is supported by research grants from the National Basic Research Program (Grant No.2004CB318110), the Natural Science Foundation of China (Grant No.60723005, 60736018, 60702024), NLPR 2008NLPRZY-2, the National Hi-Tech Research and Development Program of China (2006AA01Z193,2007AA01Z162).

\section{References}

[1] Zhang, D., Kong, W.K., You, J., Wong, M.: Online Palmprint Identification. IEEE Transactions on Pattern Analysis and Machine Intelligence 25(9), 1041-1050 (2003)

[2] Zhang, D., Shu, W.: Two Novel Characteristics in Palmprint Verification: Datum Point Invariance and Line Feature Matching. Pattern Recognition 32(4), 691-702 (1999)

[3] Duta, N., Jain, A.K., Mardia, K.V.: Matching of Palmprint. Pattern Recognition Letters 23(4), 477-485 (2001)

[4] Wu, X.Q., Wang, K.Q., Zhang, D.: Palmprint Recognition using Directional Line Energy Feature. In: Proceedings of the 17th ICPR, vol. 4, pp. 475-478 (2004)

[5] Zhang, L., Zhang, D.: Characterization of Palmprints by Wavelet Signatures via Directional Context Modeling. IEEE Trans on SMC-B 34(3), 1335-1347 (2004)

[6] You, J., Kong, W.K., Zhang, D., Cheung, K.: On Hierarchical Palmprint Coding with Multi-features for Personal Identification in Large Databases. IEEE Transactions on Circuit Systems for Video Technology 14(2), 234-243 (2004)

[7] Kong, W.K., Zhang, D.: Feature-Level Fusion for Effective Palmprint Authentication. In: Zhang, D., Jain, A.K. (eds.) ICBA 2004. LNCS, vol. 3072, pp. 761-767. Springer, Heidelberg (2004) 
[8] Kong, W.K., Zhang, D.: Competitive Coding Scheme for Palmprint Verification. In: Proceedings of the 17th ICPR, vol. 1, pp. 520-523 (2004)

[9] Sun, Z.N., Tan, T.N., Wang, Y.H., Li, S.Z.: Ordinal Palmprint Representation for Personal Identification. In: Proceedings of CVPR 2005, vol. 1, pp. 279-284 (2005)

[10] Han, Y.F., Sun, Z.N., Tan, T.N.: Palmprint Recognition Based on Directional Features and Graph Matching. In: Lee, S.-W., Li, S.Z. (eds.) ICB 2007. LNCS, vol. 4642, pp. 1164-1173. Springer, Heidelberg (2007)

[11] Koenderink, J., van Doorn: Generic neighbourhood operators. IEEE Transactions on Pattern Analysis and Machine Intelligence 14, 597-605 (1992)

[12] Daugman, J.G.: Two-dimensional spectral analysis of cortical receptive field profiles. Vision Research 20, 847-856 (1980)

[13] Florack, L.: Image Structure. Kluwer Academic Publishers, Dordrecht (1997)

[14] Tuzel, O., Porikli, F., Meer, P.: Region covariance: A fast descriptor for detection and classification. In: Leonardis, A., Bischof, H., Pinz, A. (eds.) ECCV 2006. LNCS, vol. 3952, pp. 586-600. Springer, Heidelberg (2006)

[15] PolyU Palmprint Database, http: / /www . comp.polyu. edu.hk/ biometrics /

[16] CASIA Palmprint Database, http: / / www.cbsr.ia.ac.cn/english/Palmprint\%20Databases.asp

[17] Daugman, J., Williams, G.: A Proposed Standard for Biometric Decidability. In: Proceedings of CardTech/ SecureTech Conference, Atlanta, GA, pp. 223-234 (1996) 